\title{
Production, Commissioning and First Data of the LHCb Silicon Tracker
}

\begin{abstract}
We give here a short review of the LHCb Silicon tracker, the main points of the module production and quality control, followed by the commissioning of the detector. Problems that were found during production or commissioning are described and the first performance assessment of the installed detector with "beam data" is given.
\end{abstract}

Key words: LHCb, silicon, tracker, detector PACS: 07.77.Ka, 29.40.Wk,

\section{Introduction}

The LHCb detector [2] at the Large Hadron Collider is dedicated to study the physics in the decay of b-flavoured hadrons. It is designed as a single arm forward spectrometer adapted to the angular distribution of the $b \bar{b}$ pairs which are produced predominantly at low polar angles. The $\mathrm{LHCb}$ Silicon Tracker consists of two subdetectors: the TT-Station [3] (Tracker Turicensis) which forms a 


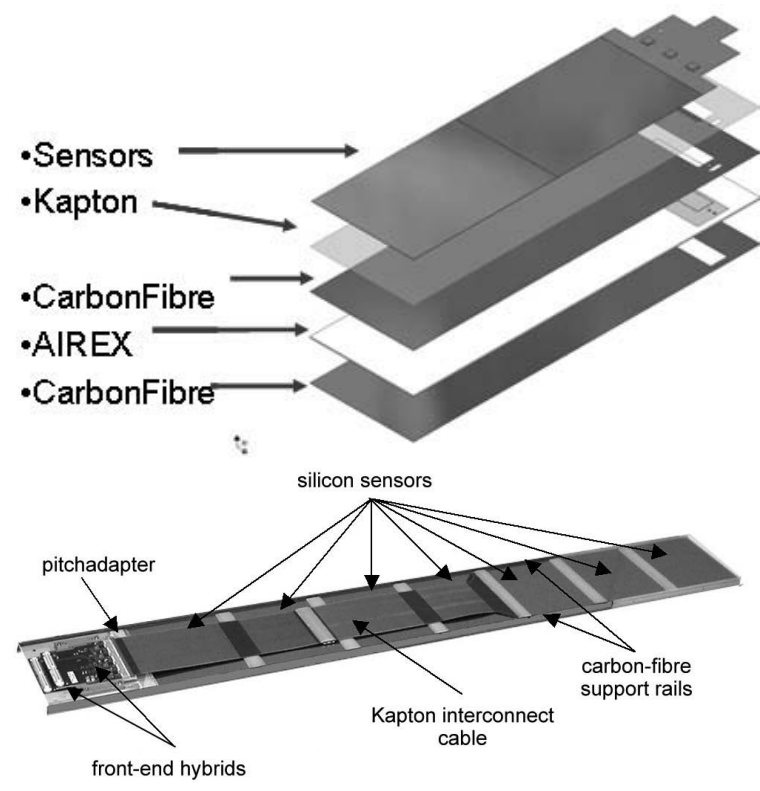

Fig. 1. Schematics of the detector modules of the Inner Tracker (top) and the TT-Station (bottom).

tracking station in front of the 4 Tm dipole bending magnet, and the Inner Tracker [4] that covers the high particle density region near the beampipe in three tracking stations downstream of the magnet. The outer regions of these three tracking stations are covered by the Outer Tracker with straw tubes. The $\mathrm{LHCb}$ tracking system is completed with the silicon vertex detector (VELO).

\section{Silicon Tracker Design}

The Silicon Tracker project consists of the TTStation and the Inner Tracker, both employing silicon strip detectors. Each Silicon Tracker station consists of four detection layers where the inner two layers are arranged with a $\pm 5^{\circ}$ stereo angle. The TT-Station, a single tracking station consisting of four layers, is housed in a single large box while each of the three Inner Tracker stations consists of four individual detector boxes surrounding the beam pipe. The detector boxes each house the 4 detection layers of a station. The boxes provide electrical and thermal insulation. The sensors are silicon strip detectors with pitches of $183 \mu \mathrm{m}$ and
$198 \mu \mathrm{m}$, for the TT-Station and the Inner Tracker, respectively. Up to four silicon sensors are bonded together in the TT-Station to form a single readout strip with an effective sensor lengths ranging from $9.4 \mathrm{~cm}$ to $37.2 \mathrm{~cm}$. All the readout hybrids for the TT-Station are located outside of the acceptance. The inner readout sectors are connected with up to $56 \mathrm{~cm}$ long Kapton interconnect cables to the respective readout hybrid. In the Inner Tracker single-sensor modules are employed in the central boxes with a length of $10.8 \mathrm{~cm}$ and two sensor modules with $21.6 \mathrm{~cm}$ sensor length in the side boxes. The sensors used for the one and two-sensor Inner Tracker modules have a thickness of $320 \mu \mathrm{m}$ and $410 \mu \mathrm{m}$, respectively. In the TT-Station $500 \mu \mathrm{m}$ thick sensors are needed to ensure good signalover-noise ratio because of the larger load capacitance to the preamplifier resulting from the long readout strips and interconnect cables. Schematics of the detector modules are depicted in Figure 1. The Silicon Tracker covers a total area of $12.2 \mathrm{~m}^{2}$ with $270 \mathrm{k}$ readout channels, distributed over 280 readout sectors in the TT-Station and 336 modules in the Inner Tracker.

The highest radiation dose in the Silicon Tracker is collected by the innermost part of the TTStation and amounts to about $10^{14} 1 \mathrm{MeV}$ neutron equivalent $/ \mathrm{cm}^{2}$ in ten years of LHCb operation [5]. The analog signals from the radiation hard "Beetle" readout chip [6] are sent via up to $9 \mathrm{~m}$ long copper cables to the service boxes $[7,8]$ located just outside of the acceptance of the experiment. There the data is digitised and converted into optical signals that are then tranmitted via optical fibres to the counting house located in a radiation free zone behind a shielding wall in the cavern.

\section{Production}

During module production, the sensors were positioned using jigs with mechanical stops, relying on the precision of the cutting edges of the silicon sensors that was specified to be better than \pm $20 \mu \mathrm{m}$. The sensors were then held in place by vacuum for the gluing of the modules. After bonding of the readout hybrid and sensors, the modules were 
then tested for bias voltage stability and subjected to temperature cycling between $-5^{\circ} \mathrm{C}$ and $+40^{\circ} \mathrm{C}$ $\left(+5^{\circ} \mathrm{C}\right.$ and $+25^{\circ} \mathrm{C}$ for the TT-Modules $)$ and electrical burn in for up to $48 \mathrm{~h}$. Two Inner Tracker modules out of the production failed during the electrical burn in, which was traced to a weak insulation on the hybrid side and the use of conductive glue. The glue was then replaced by nonconductive Araldite for the rest of the production. Open and shorted channels present a different load capacitance to the pre-amplifier compared to properly connected readout channels, affecting channel noise and pulseshape. They are therefore detected during the readout test by either the channel noise or the pulse height observed when using the internal beetle test pulse to inject charge into the pre-amplifier. Pinholes were detected by subjecting the unbiased detector to (infrared-)light. This then leaves a signature of low noise in channels with pinholes due to the saturation of the pre-amplifier from the photo current.

\section{Commissioning and first Data}

The detector has been installed and put into operation in spring and summer 2008. Several problems summarised below were observed and subsequently fixed. The readout electronics was not previously tested for all different load scenarios as encountered in the final detector. Additional capacitors of $1 \mathrm{mF}$ needed to be added to prevent occasional oscillations, in power supply lines of the service boxes that were subjected to the biggest load, occuring in the TT-Station in the quadrant where the longest cables are used. These long cables between the modules and the service boxes also required fine adjustment of voltage dividers that are in front of the ADC used for the monitoring of the analog supply voltage of the readout chips. During the production tests of the service boxes housing the digitiser cards and the control cards in the laboratory, there were no actual readout hybrids connected to each input. This resulted in the fact that some faults in individual $\mathrm{I}^{2} \mathrm{C}$-bus lines and missing or badly soldered noise filters at the digitiser board escaped discovery prior to their installation in the experimental hall. For the TTStation where the service boxes are easily accessible, the previously mentioned problems were immediately repaired, while for the Inner Tracker the repair has been postponed to the winter shutdown $2008 / 2009$ as the service boxes are difficult to access. Despite these not yet fixed problems, $97 \%$ of the Inner Tracker were functional during the 2008 running. Of the TT-Station, $99 \%$ were fully functioning but 2 modules showed low breakdown voltage in the silicon sensor and will also be replaced in the winter shutdown.

Performance studies in Summer 2008 have been performed with two different type of data. The first set has been collected during the LHC synchronisation tests, when the injection beam was dumped onto a beam dump in the transfer line located about $300 \mathrm{~m}$ behind the $\mathrm{LHCb}$ detectors. The other data results from cosmic triggers. Despite the horizontal orientation of $\mathrm{LHCb}$, several hundred cosmic events containing at least one part that crosses an Inner Tracker detector box have been found in 1.1 million cosmic triggers. Fortyfive of these events contain track segments that cross neighbouring stations and two events gave a track that could be reconstructed in all three Inner Tracker stations. One of the latter events is shown in Figure 2: Ten out of twelve possible hits

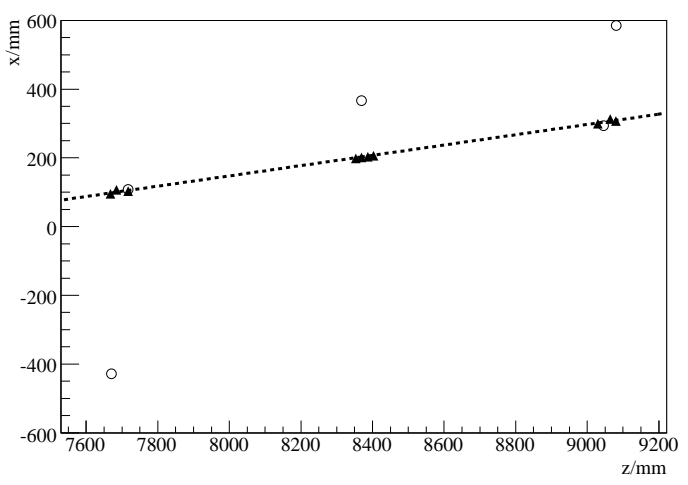

Fig. 2. Reconstructed track of a cosmic particle in the Inner Tracker in $\mathrm{x}-\mathrm{z}$ projection. The triangles are hits assigned to the track. The open circles show unassigned hits in the event.

are included in the reconstructed track, one hit is just outside of the search window while the last 
one is missing. This figure also allows the level of detector noise to be judged, as the number of noise hits is very low. The hit residuals of all reconstructed track segments that cross at least two Inner Tracker stations are displayed in Figure 3. These track residuals can be used to get a very

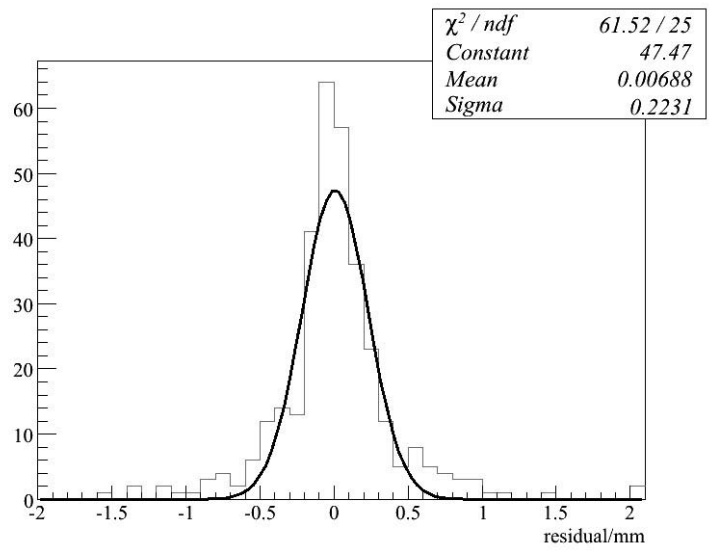

Fig. 3. Residuals of reconstructed track segments of a cosmic ray particles in the Inner Tracker that cross detector boxes in at least two stations.

first glimpse of the alignment of the detector. Using the nominal geometry in the reconstruction, the track residuals have an RMS of $310 \mu \mathrm{m}$. Including information from a partial survey this is reduced to $270 \mu \mathrm{m}$. Using more relative alignment information from events taken during the synchronisation tests, this is further reduced to $220 \mu \mathrm{m}$. These values are reasonable given the known tolerances of the survey and the positioning of the detector [10] which are estimated to $50 \mu \mathrm{m}$ of the modules relative to each other within a detector box and about $1 \mathrm{~mm}$ for the box positioning.

These beam dumps during the LHC synchronisation tests resulted in events in LHCb with a particle multiplicity of about 20 times higher than during nominal running. This very high multiplicity complicates the reconstruction of individual tracks in the Silicon Tracker. However overall correlations plots have been used to extract relative alignment information.

The four detection layers in the TT-Station are not sufficient to reconstruct track segments. Here alignment studies using the data from the LHC synchronisation test were done using extrapolated
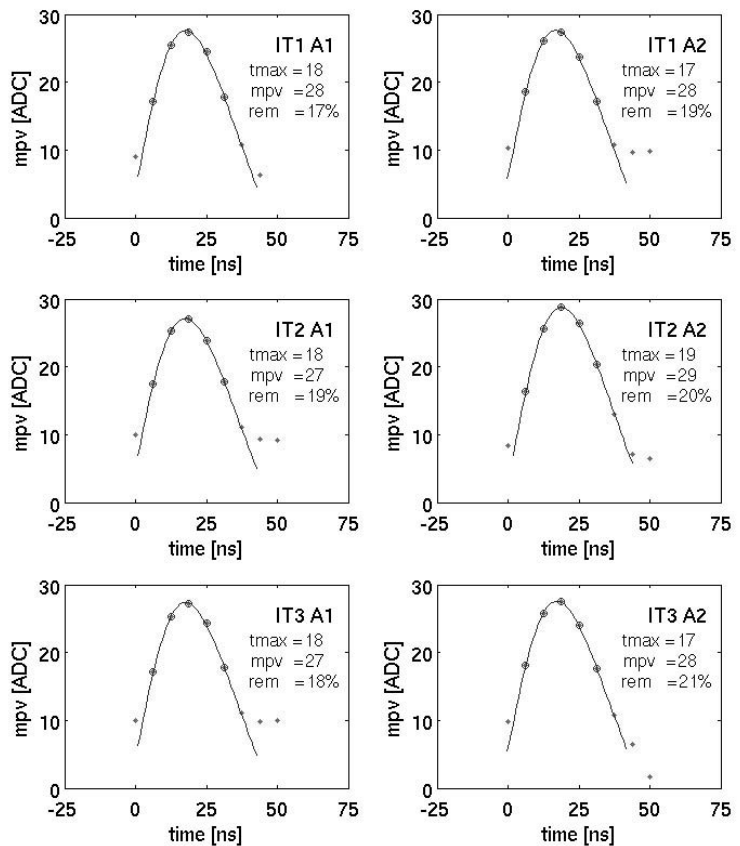

Fig. 4. The most probable value (mpv) of the sampled charge deposition in the silicon for different timing settings, resulting in a pulseshape. IT1-IT3 refer to the three different Inner Tracker stations and A1,A2 to different regions within a station. The events were recorded when the LHC injection beam was dumped in the transfer line during LHC synchronisation tests.

tracks from the VELO and hits in the TT-Station. The RMS of the hits associated to an extrapolated VELO track is about $500 \mu \mathrm{m}$ with $300 \mu \mathrm{m}$ expected from simulations for perfect alignment. The offset varies between $150-300 \mu \mathrm{m}$ for the different Layers of the TT-Station, demonstrating a very good mechanical alignment. The LHC synchronisation test events were extremely useful for the internal time alignment of the different parts of the Silicon Tracker and a first determination of the signal-over-noise ratio $(\mathrm{S} / \mathrm{N})$. In Figure 4 reconstructed pulseshapes from changing the sampling time of the Inner Tracker front end chip are shown. The peak time differs slightly for different parts in the detector that have different physical location along the particles flight path and different cable lengths, which both need to be adjusted for by choosing the optimal clock delays. The optimal delay can easily be determined to about a 
precision of $1 \mathrm{~ns}$ from the pulseshapes. In addition, the maximum of this plot gives the most probable value (mpv) of the charge measured in the sensor. Together with the noise measured for the detector modules, this results in an $\mathrm{S} / \mathrm{N}$ for the detector of about 14 for the Inner Tracker and TT-Station modules which is only about $10 \%$ lower than in the original expectation [9].

\section{Summary}

The LHCb Silicon Tracker has been installed in the $\mathrm{LHCb}$ detector and put successfully into operation in summer 2008. Cosmic ray particles and events from LHC beam dump during synchronisation tests were used for first alignment studies, the determination of the $\mathrm{S} / \mathrm{N}$ in the detector, as well as the internal time alignment. The relative alignment and $\mathrm{S} / \mathrm{N}$ are close to expectation.

\section{References}

[1] Production, Commissioning and First Data of the LHCb Silicon Tracker, LHCb-2008-062.

[2] LHCb Coll., LHCb Technical Proposal, CERN/LHCC 98-4.

[3] LHCb Coll., LHCb Reoptimized Detector, Design and Performance, CERN/LHCC 2003-030.

[4] LHCb Coll., LHCb Inner Tracker, CERN/LHCC 2002029 .

[5] Expected Particle Fluences and Performance of the $\mathrm{LHCb}$ Trigger Tracker, M . Siegler, F. Lehner, M. Needham, O. Steinkamp, LHCb-2004-070.

[6] The

Beetle Reference Manual,// http://wwwasic.kip.uniheidelberg.de/lhcb/Publications/BeetleRefMan_v1_3.pdf.

[7] An Optical Readout System for the LHCb Silicon Tracker, A. Vollhardt, PhD thesis, Universität Zürich (2005).

[8] Design and development of the Control Board for the LHCb Silicon Tracker, D. Esperante, LHCb-2007-153.

[9] Test-beam measurements on prototype ladders for the LHCb TT station and Inner Tracker, M. Agari et.al., LHCb-2003-082; Layout and Expected Performance of the LHCb TT Station, J. Gassner,M. Needham, O. Steinkamp, LHCb-2003-140.
[10] Inner Tracker Survey Strategy, G. Conti and al., LHCb-2008-068 IT Survey measurements : Analysis and Implementaiton in the LHCb Software, G.Conti, F.Blanc, LHCb-2008-069. 